\title{
OPTICAL BACKGROUND FROM GALAXIES
}

\author{
M. Kalinkov and I. Kuneva \\ Department of Astronomy \\ Bulgarian Academy of Sciences \\ 72 Lenin Blvd. \\ Sofia 1784, Bulgaria
}

ABSTRACT. The optical brightness of the sky due to galaxies is determined up to 15.7 Zwicky magnitude and to $B=15^{m}$.

\section{INTRODUCTION}

Some years ago we initiated a project known as the Metacatalog of galaxies. The early state of the project is given by Kalinkov and Kuneva (1982) and Kalinkov (1982). The Metacatalog contains information for galaxies, clusters of galaxies, counts of galaxies and references.

Now we have a merged catalog of galaxies in which the main catalogs of galaxies are incorporated, e.g., Zwicky et al. (1961-1965), Nilson (1973), Vaucouleurs et al. (1976), Lauberts (1982), Huchra et al. (1983), Fairall and Jones (1988). Many lists of galaxies are also included in our catalog.

Here we use this catalog to compute the optical brightness of the sky due to galaxies. Two cases are studied: all Zwicky galaxies $(27,841)$ and all galaxies up to $B_{T}=15.0(14,361)$.

An attempt to remove the influence of absorption in our Milky Way is made.

\subsection{Zwicky Galaxies}

We use the original magnitude of the Zwicky catalog without any correction.

Assuming a $\csc (b)$ law for the galactic extinction, a standard procedure for solution of

$$
\log N(b)=\log N_{0}+0.6 A_{V} \csc (b)
$$

is applied. $\mathrm{N}(b)$ is the number of galaxies (per square degree) observed at galactic latitude $b$, and $A_{V}$ is the extinction coefficient. The galactic latitude in (1) is $b=\sin ^{-1}((\sin b+\sin$ $(b+\Delta b)) / 2)$, which halves the corresponding zones of $\Delta b$.

The coefficients are given in Table 1.

\begin{tabular}{ccccc}
\multicolumn{3}{c}{ Table 1. Coefficients of the csc $(b)$ Law } \\
\hline \hline \multicolumn{4}{c}{$\Delta b=5^{\circ}$} & \multicolumn{2}{c}{$\Delta b=10^{\circ}$} \\
\hline & $b>0$ & $b \leq 0$ & $b>0$ & $b \leq 0$ \\
$A_{V}$ & 0.256 & 0.225 & 0.322 & 0.316 \\
$\log N_{0}$ & 0.497 & 0.451 & 0.573 & 0.558 \\
\hline
\end{tabular}

The most reliable extinction coefficient is $A_{V}=0.256$, the Hubble's value. Therefore, the Zwicky catalog may be used to derive $A_{V}$ (cf. Vaucouleurs and Buta, 1983).

All Zwicky magnitudes are corrected for galactic extinction. The number of galaxies which could not have been observed due to absorption is determined in accordance with the observed luminosity function and the latter are added in the magnitude range ([15.7 - $A_{V}$ $\csc (b)] \div 15.7)$. Similar corrections are made for regions outside the scope of the Zwicky catalog. 
Table 2. Optical Brightness ( $m a g \operatorname{arcsec}^{-2}$ ), due to Galaxies up to $m_{Z w}=15.7$ and $B_{T}=15.0$

\begin{tabular}{lrrrrrrr}
\multicolumn{7}{c}{ Galaxies up to $m_{Z w}=15.7$ and $B_{T}=15.0$} \\
\hline \hline$m_{Z w}, B_{T}$ & 12.0 & 13.0 & 14.0 & 14.5 & 15.0 & 15.5 & 15.7 \\
\hline$b$ & & & & & & & \\
$85^{\circ}-90^{\circ}$ & 31.2 & 31.1 & 30.8 & 30.7 & 30.5 & 30.3 & 30.2 \\
& 31.0 & 30.9 & 30.7 & 30.6 & 30.2 & & \\
$80-85$ & 31.4 & 31.3 & 31.1 & 31.0 & 31.0 & 30.8 & 30.7 \\
& 31.2 & 31.1 & 31.0 & 30.9 & 30.7 & & \\
$75-80$ & 31.3 & 31.0 & 30.9 & 30.8 & 30.7 & 30.6 & 30.5 \\
& 31.1 & 30.9 & 30.7 & 30.7 & 30.4 & & \\
$70-75$ & 31.6 & 31.2 & 31.0 & 30.9 & 30.8 & 30.5 & 30.4 \\
& 31.4 & 31.1 & 30.9 & 30.8 & 30.6 & & \\
$65-70$ & 31.8 & 31.5 & 31.3 & 31.2 & 31.0 & 30.8 & 30.7 \\
& 31.6 & 31.4 & 31.1 & 31.0 & 30.8 & & \\
$60-65$ & 32.2 & 31.9 & 31.6 & 31.6 & 31.4 & 31.1 & 31.0 \\
& 32.1 & 31.8 & 31.5 & 31.4 & 31.1 & & \\
$55-60$ & 31.8 & 31.6 & 31.4 & 31.3 & 31.1 & 31.1 & 30.7 \\
& 31.8 & 31.6 & 31.3 & 31.2 & 31.0 & & \\
Mean & 31.6 & 31.4 & 31.1 & 31.1 & 30.9 & 30.7 & 30.6 \\
st. dev. & 0.3 & 0.3 & 0.3 & 0.3 & 0.3 & 0.3 & 0.3 \\
Mean & 31.4 & 31.2 & 31.0 & 30.9 & 31.0 & & \\
st. dev. & 0.4 & 0.4 & 0.3 & 0.3 & 0.5 & & \\
\hline
\end{tabular}

Table 2 contains the results. In this table, the first rows refer to the optical background from galaxies, up to $m_{Z w}=15.7$.

\subsection{Galaxies up to $B=\mathbf{1 5 . 0}$}

Some relations between $B_{T}(\mathrm{RC} 2)$ and magnitudes from other catalogs have been found. These relations are used for transformation into the $B_{T}$ system. For this sample of galaxies $A_{V}=$ 0.172 (for $b>0$ ) and 0.155 (for $b \leq 0$ ). The results for the $B_{T}$ background are given by the second row of numbers in Table 2.

Acknowledgments - We express our gratitude to D. Stefanova and H. Macrelov for their help.

\section{REFERENCES}

de Vaucouleurs, G., and Buta, R. 1983, A. J., 88, 939.

de Vaucouleurs, G., de Vaucouleurs, A., and Corwin, H. G. 1976, Second Reference Catalogue of Bright Galaxies (University of Texas Press).

Fairall, A. P, and Jones, A. 1988, Publ. Dept. Astronomy, Univ. Cape Town, No. 10.

Huchra, J., Davis, M., Latham, D., and Tonry, J. 1983, Ap. J. Suppl., 52, 89.

Kalinkov, M. 1982, in Automated Data Retrieval in Astronomy, ed. C. Jaschek and W. Heintz (D. Reidel: Dordrecht), 259.

Kalinkov, M., and Kuneva, I. 1982, in Automated Data Retrieval in Astronomy, ed. C. Jaschek and W. Heintz (D. Reidel: Dordrecht), 261.

Lauberts, A. 1982, The ESO/Uppsala Survey of the ESO(B) Atlas (European Southern Observatory).

Nilson, p. 1973, Uppsala General Catalogue of Galaxies (Uppsala).

Zwicky, F., Herzog, E., Wild, P., Karpowicz, M, and Kowal, C. T. 1961-1968, Catalogue of Galaxies and of Clusters of Galaxies, 1-6 (California Institute of Technology). 